\title{
Laser-induced forward transfer of liquids: Study of the droplet ejection process
}

\author{
M. Colina, M. Duocastella, J. M. Fernández-Pradas, P. Serra, ${ }^{a)}$ and J. L. Morenza \\ Departament de Física Aplicada i Òptica, Universitat de Barcelona, Martí i Franquès 1, E-08028 Barcelona, \\ Spain
}

(Received 7 July 2005; accepted 9 March 2006; published online 3 May 2006)

\begin{abstract}
Laser-induced forward transfer (LIFT) is a laser direct-write technique that offers the possibility of printing patterns with a high spatial resolution from a wide range of materials in a solid or liquid state, such as conductors, dielectrics, and biomolecules in solution. This versatility has made LIFT a very promising alternative to lithography-based processes for the rapid prototyping of biomolecule microarrays. Here, we study the transfer process through the LIFT of droplets of a solution suitable for microarray preparation. The laser pulse energy and beam size were systematically varied, and the effect on the transferred droplets was evaluated. Controlled transfers in which the deposited droplets displayed optimal features could be obtained by varying these parameters. In addition, the transferred droplet volume displayed a linear dependence on the laser pulse energy. This dependence allowed determining a threshold energy density value, independent of the laser focusing conditions, which acted as necessary conditions for the transfer to occur. The corresponding sufficient condition was given by a different total energy threshold for each laser beam dimension. The threshold energy density was found to be the dimensional parameter that determined the amount of the transferred liquid per laser pulse, and there was no substantial loss of material due to liquid vaporization during the transfer. (0) 2006 American Institute of Physics. [DOI: 10.1063/1.2191569]
\end{abstract}

\section{INTRODUCTION}

Recent advances in genetic and molecular biology have prompted the development of highly integrated and miniaturized biosensors. Such devices are powerful tools for application in cell biology research, medical diagnostics, therapeutic studies, detection of pathogenic agents, and tissue engineering. Although pattern-transfer techniques such as photolithography are adequate for the large-scale production of miniaturized biosensors, they are not versatile enough for rapidprototyping applications. Direct-write technologies represent an interesting alternative when a fast and inexpensive production of the operative prototype is required. However, the more conventional direct-write techniques use pins and printing headers ${ }^{1,2}$ that, due to their size and arrangement, restrict both the spatial resolution and the versatility of such techniques. Laser-induced forward transfer (LIFT) is a noncontact direct-writing technique that has emerged as an alternative for rapid-prototyping applications due to its capacity to overcome various difficulties associated with conventional direct-write techniques. In addition, LIFT allows the transfer of a wide range of materials, in air and at room temperature. All of these characteristics make LIFT suitable for the preparation of integrated biosensors through a unique process that includes several serial steps, and, consequently, a significant reduction in the production time of the biosensors can be achieved.

In the LIFT technique, a focused laser beam removes a tiny amount of the material to be transferred from a precursor film deposited on a transparent support. The material is then deposited on a receptor substrate placed alongside the pre-

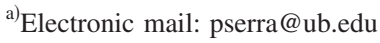

cursor film. The short separation between the precursor film and the receptor substrate, and the reduced dimensions of the focused laser beam provide this technique with the desired spatial resolution. Since its development by Bohandy et al., ${ }^{3}$ the feasibility of LIFT for the deposition of inorganic materials in a solid state has been repeatedly demonstrated. ${ }^{4-6}$ In this case, the heating caused by the laser in the precursor film leads to the vaporization of the material, which recondenses in the receptor substrate following the transfer. Under these conditions, the transfer of biomolecules is clearly not possible due to the irreversible damage to the biomaterial caused by a direct interaction with the laser radiation. The possibility of using LIFT to transfer biomolecules in a solution without a decomposition of the transferred biomolecules was reported by Wu et al. ${ }^{7}$ When applying LIFT to the transfer of biomolecules in a solution, the laser pulse causes an expulsion of a part of the liquid, which is then deposited on the substrate. In this case, the solvent acts as a transport vector and at the same time prevents the decomposition of the biomolecules. Accordingly, various biological materials, including proteins, ${ }^{8-10}$ DNA, ${ }^{11-13}$ and cells ${ }^{14-16}$ have been transferred without a significant damage.

Although the feasibility of using LIFT to transfer microarrays of biomolecules in a solution has been considerably explored, studies of the dependence of the properties of the transferred droplets on the different technological parameters are still insufficient to gain an in-depth understanding of the transfer process. ${ }^{8,9,13,17,18}$ The aim of our work is to carry out a study that contributes to fill this void. In this study we analyze the morphological properties of droplets transferred using LIFT at different laser pulse energies and laser beam dimensions. The liquid mixture chosen to per- 
form the study is suitable to act as a solvent for biomolecules. The results of this study should not only provide a better understanding of the transfer process but should also allow an optimal set of technological parameters to be determined for the generation of microarrays with suitable features for biosensor-related applications.

\section{EXPERIMENT}

A pulsed Nd:YAG (yttrium aluminum garnet) laser beam (355 $\mathrm{nm}$ wavelength, $10 \mathrm{~ns}$ pulse duration) was used to deposit microarrays using LIFT to transfer microdroplets of a solution. The transferred solution consisted of a mixture of water and glycerol, both at a concentration of 50\% $(v / v)$, and a surfactant [sodium dodecyl sulphate (SDS)] dissolved at a concentration of $2.0 \mathrm{mg} / \mathrm{ml}$. The support on which the liquid film was spread consisted of a transparent glass slide coated with a titanium thin film, which was deposited by thermal evaporation and had a thickness of around $60 \mathrm{~nm}$. It is important to note that the solution used is transparent to laser radiation. For this reason, a titanium coating was incorporated as an absorbing layer. The use of absorbing layers of materials such as $\mathrm{Au},{ }^{19} \mathrm{Ti},{ }^{9,13,19}$ and $\mathrm{Ag}^{20}$ has been tested with positive results in the transfer of biomolecules and cells. A volume of $20 \mu \mathrm{l}$ of the prepared solution was spread on the titanium thin film with a blade coater. The liquid film thickness, estimated through the measurement of the film weight, was approximately $7 \mu \mathrm{m}$. The system formed by the titanium-coated slide and the solution thin film has usually been referred to as a ribbon. ${ }^{21}$ The solution was transferred to a commercially available glass treated with poly- $L$-lysine, a conventional receptor substrate for microarrays production. The ribbon was placed parallel to the receptor substrate, with the liquid film facing the poly- $L$-lysine-treated surface at a distance of $100 \mu \mathrm{m}$. The ribbon-substrate system was laid on an $x y z$ translation stage that permitted the precise translation of the ribbon-substrate system with respect to the laser beam. The transfer was carried out in such a way that each single laser pulse was responsible for the deposition of a unique droplet of the solution onto the substrate. After the deposition of each droplet, the stage was displaced at a distance of $150 \mu \mathrm{m}$, and another droplet was transferred at another substrate position until each single row in the microarray was completed. The laser beam displayed an elliptical Gaussian intensity distribution and was focused on the titanium coating with a $15 \times$ microscope objective. The beam dimensions were varied by modifying the distance between the titanium coating and the system focus; to obtain larger beam dimensions the ribbon-substrate system was displaced above the plane of focus. Five microarrays of the described solution were deposited. For each one, the beam size was fixed and the laser pulse energy was varied from one row to another. The major and minor radii of the elliptical beam used in each microarray are presented in the first two columns of Table I. All of the microarrays contained nine rows of 80 droplets. Once deposited, the microarrays were observed under an optical microscope to check the properties of the droplets and
TABLE I. Major $\left(\omega_{x}\right)$ and minor $\left(\omega_{y}\right)$ laser beam radii, transfer energy threshold $\left(E_{\min }\right)$, and parameters $\left(K\right.$ and $\left.E_{0}\right)$ of the five microarrays prepared using LIFT, obtained from the linear fits using Eq. (1).

\begin{tabular}{ccccc}
\hline \hline $\begin{array}{c}\omega_{x} \\
(\mu \mathrm{m})\end{array}$ & $\begin{array}{c}\omega_{y} \\
(\mu \mathrm{m})\end{array}$ & $\begin{array}{c}\mathrm{E}_{\text {min }} \\
(\mu \mathrm{J})\end{array}$ & $\begin{array}{c}K \\
(\mathrm{pL} / \mu \mathrm{J})\end{array}$ & $\begin{array}{c}E_{0} \\
(\mu \mathrm{J})\end{array}$ \\
\hline 11 & 11 & 1.1 & 1.4 & 0.1 \\
31 & 25 & 3.6 & 3.2 & 1.2 \\
51 & 36 & 6.2 & 3.3 & 3.8 \\
73 & 47 & 11 & 3.1 & 6.8 \\
95 & 64 & 20 & 3.2 & 11.5 \\
\hline \hline
\end{tabular}

to measure their dimensions. Likewise, the spots produced by the laser beam on the titanium layer were also evaluated by optical microscopy.

\section{RESULTS AND DISCUSSION}

Optical microscopy images of a representative portion of four of the obtained microarrays are shown in Fig. 1. Each microarray was deposited with a different sized laser beam, and the laser pulse energy reported in each row corresponds to an average of the energies of all the pulses used in that row. It is a common trend in all microarrays that the droplet dimensions increase with the laser pulse energy. Therefore, at fixed laser beam dimensions, the use of higher pulse energies results in the release of larger amounts of material from the liquid film. However, there is a limit to how far a droplet diameter can be minimized by setting lower laser pulse energy values, as would be desirable for microarray miniaturization, since there is always a minimum droplet di-

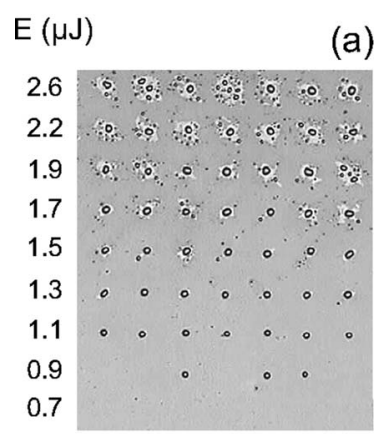

(c)

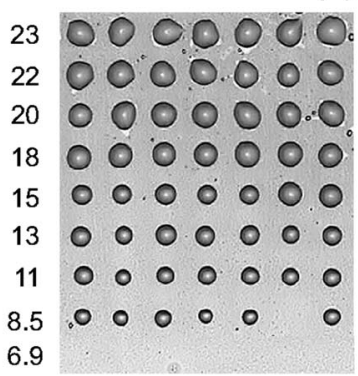

$E(\mu J)$

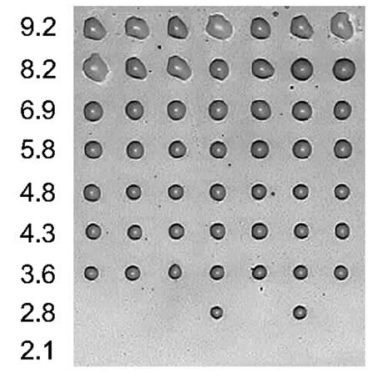

(d) (b)

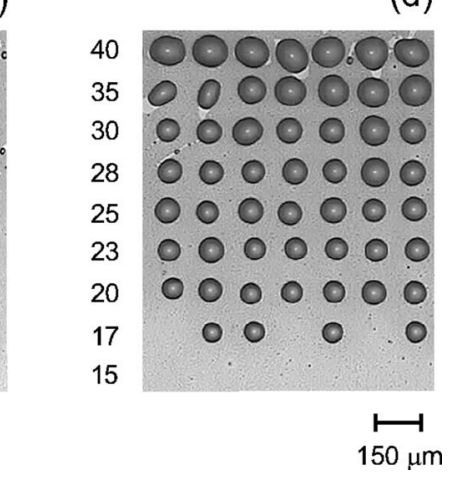

FIG. 1. Optical microscopy images of the microarrays prepared at four different beam sizes: (a) $\omega_{x}=\omega_{y}=11 \mu \mathrm{m}$; (b) $\omega_{x}=31 \mu \mathrm{m}, \omega_{y}=25 \mu \mathrm{m}$; (c) $\omega_{x}=73 \mu \mathrm{m}, \omega_{y}=47 \mu \mathrm{m}$; and (d) $\omega_{x}=95 \mu \mathrm{m}, \omega_{y}=64 \mu \mathrm{m}$. All the droplets in each row were transferred at the laser pulse energy indicated in the image. 
ameter that can be achieved for a fixed set of laser beam dimensions. This can be clearly observed in the last rows corresponding to the two lower energies in each microarray: the penultimate row always contains gaps in the transfer, and no droplets can be detected in the last row in any of the cases. This is due to the fact that below a certain laser pulse energy threshold no material can be transferred. This threshold would correspond to the average energy $E_{\min }$ of the pulses used to print the penultimate rows (Table I), and the gaps in the transfer can be attributed to individual laser pulses with energies just slightly below the threshold. Furthermore, a comparison of the last rows of all the microarrays indicates that the smaller the dimensions of the laser beam, the smaller the diameter of the transferred droplets. In this particular case, the minimum droplet diameters obtained with no transfer gaps and good reproducibility are approximately $30 \mu \mathrm{m}$ [Fig. 1(a), $E=1.1 \mu \mathrm{J}$ ], a value well below the usual $100 \mu \mathrm{m}$ diameter obtained with conventional microarray spotters. ${ }^{22,23}$ A modification of the two technological parameters analyzed in this study affects not only the dimensions of the transferred droplets but also their morphology. Thus, laser pulse energies just above the transfer threshold generate droplets that are all well defined, rounded, and quite uniform, irrespective of the laser beam dimensions. However, as the energy increases, the quality in terms of definition and uniformity of the droplets decreases. At the smallest laser beam dimensions [Fig. 1(a)], energies higher than $1.3 \mu \mathrm{J}$ lead to droplets that are more irregular and present small satellites and a significant splashing. With the other focusing conditions, although no splashing is observed, an increased laser pulse energy leads to droplets that also display irregular shapes [Figs. 1(b)-1(d)] and, in some cases, sporadic satellites can be detected [Figs. 1(c) and 1(d)]. These behaviors may be explained in terms of the kinetic energy of the droplets just before their impact against the receptor substrate. At laser pulse energies just above the transfer threshold, it can be assumed that the kinetic energy of the droplets will be low enough to allow them to be gently deposited onto the substrate and that this would lead to symmetrical circular droplets. When the kinetic energy increases (higher laser pulse energies), the spreading and recoil effects just after the impact against the substrate are greater. For surfaces that are not perfectly clean, a loss of symmetry in the droplets occurs when they spread on the substrate. ${ }^{24}$ In commercially available poly- $L$-lysine slides, impurities and inhomogeneities are always present, and their effects become more evident precisely when the impact energies are high, accounting for the observed irregular shapes. At very high kinetic energies, spreading of the droplets can result in splashing. This phenomenon would explain the large number of satellites that surround the droplets corresponding to the high laser pulse energies in Fig. 1(a), where the laser beam is so highly focused that the kinetic energy transferred to the emerging droplets is probably much higher than those in the other microarrays shown in Fig. 1. It should be noted that in some cases even the central droplet is not present and appears to be broken into a set of much smaller droplets. In these cases, we cannot discount the possibility that instead of a droplet, an expanding jet of liquid was directly emitted

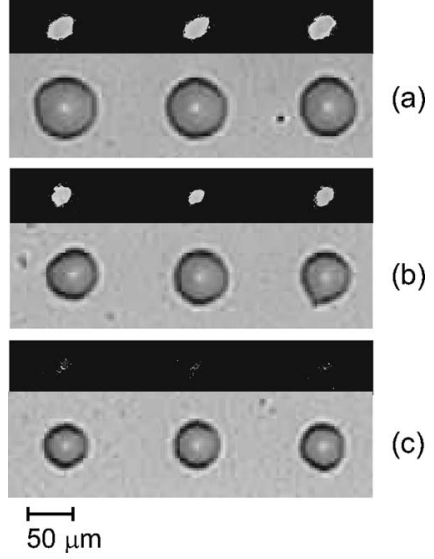

FIG. 2. Optical microscopy images of the spots on the titanium thin film and their corresponding droplets obtained with beam dimensions of $\omega_{x}$ $=31 \mu \mathrm{m}$ and $\omega_{y}=25 \mu \mathrm{m}$ for three different average laser pulse energies: (a) $6.9 \mu \mathrm{J}$, (b) $4.8 \mu \mathrm{J}$, and (c) $3.6 \mu \mathrm{J}$.

from the liquid film when the laser pulse impinged upon it. ${ }^{25}$ Finally, the isolated and sporadic satellites in Figs. 1(c) and 1(d) could be due to secondary drops produced during the recoil. ${ }^{24}$ In summary, all these considerations indicate that the optimum working conditions correspond to laser pulse energies slightly higher than the transfer threshold, where uniform, circular droplets, with neither splashing nor satellites, can be obtained. Good control of droplet dimensions can be achieved through the simultaneous variation of the laser pulse energy and laser beam focusing conditions.

Transmission optical microscopy images of the spots on the titanium thin film are shown in Fig. 2. Under each spot an optical microscopy image of its respective deposited droplet is also shown. All the spots belong to the microarray prepared with a beam size of $31 \mu \mathrm{m}$ major radius and $25 \mu \mathrm{m}$ minor radius. These images reveal that in all the cases the lateral dimensions of the droplets are considerably larger than those of the laser spots, a feature common to all of the deposited microarrays for all the analyzed laser pulse energies. The images in Figs. 2(a) and 2(b) show spots where an almost elliptical area has been completely removed from the titanium film. Although the removal of the metallic film suggests that the transferred droplets probably contain titanium residues, previous studies of DNA transfer by LIFT demonstrated that the presumptive amount of titanium in the transferred liquid, not detected after liquid drying, does not have a significant effect on the operation of the prepared biosensor. ${ }^{9,13}$ Unlike in the previous images, the spots shown in Fig. 2(c), corresponding to a lower laser pulse energy, do not exhibit a defined area of ablation. Only some cracks can be distinguished in them. Surprisingly, despite the fact that none of these spots presents a uniform ablated area, there is always a droplet transfer associated with each one of them. Two alternative transfer mechanisms might be considered to explain this result. The first mechanism, based on a thermal process, consists of heating without a significant ablation of the titanium coating due to the absorption of the laser pulse, which would lead to the vaporization of a small fraction of the solvent in contact with the titanium surface. The generated vapor would propel part of the liquid towards the recep- 


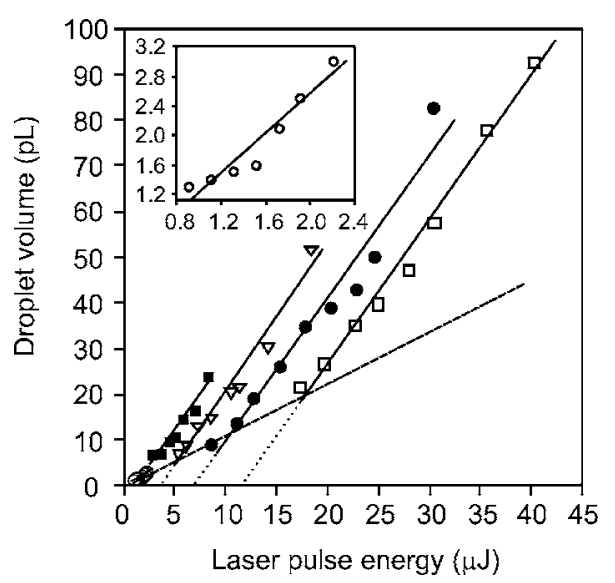

FIG. 3. Plot of the transferred droplet volume vs laser pulse energy for all beam dimensions: $(\bigcirc) \omega_{x}=\omega_{y}=11 \mu \mathrm{m} ;(\mathbb{\square}) \omega_{x}=31 \mu \mathrm{m}, \omega_{y}=25 \mu \mathrm{m} ;(\nabla)$ $\omega_{x}=51 \mu \mathrm{m}, \omega_{y}=36 \mu \mathrm{m} ;(\bullet) \omega_{x}=73 \mu \mathrm{m}, \omega_{y}=47 \mu \mathrm{m} ;(\square) \omega_{x}=95 \mu \mathrm{m}, \omega_{y}$ $=64 \mu \mathrm{m}$; and ( - and---) linear fits. The insert shows a magnification of the points corresponding to the smallest beam dimensions.

tor substrate. The second alternative mechanism, which is based on a thermal-acoustic process, ${ }^{18}$ also involves heating of the titanium coating, but in this case such heating would provoke a mechanical shock that would lead to a cracking of the titanium coating and a propulsion of a liquid droplet towards the substrate. It is important to note that the images in Fig. 2(c) correspond to a laser pulse energy just above the transfer threshold, where the smallest and most uniform droplets were obtained. Therefore, it can be concluded that not only is there no need to ablate the absorbing coating for the droplet transfer to take place, but also that the droplets with the best features are obtained under these working conditions.

In order to carry out a quantitative analysis of the obtained results, the droplet diameters were measured for all the microarrays. This was done using an appropriate software to identify the circle that best fitted the droplet perimeter seen in the optical microscopy images. Grazing incidence microscopy images of LIFT-deposited droplets of various sizes indicated that the droplet shape always corresponds to a spherical cap with a contact angle of $30 \pm 2^{\circ}$. Accordingly, the average volume of the droplets was obtained by the calculation of the volume of a spherical cap with the same diameter as that of the corresponding droplet. The standard deviation for these volumes was found to be about $20 \%$ in the case of the uniform droplets transferred at energies close to the transfer threshold and about $25 \%$ in the case of the more irregular droplets transferred at higher energies. These levels of error in reproducibility are comparable to those found with the same technique for the transfer of similar liquids. ${ }^{18}$

A graph of the average volume of the transferred droplets versus the laser pulse energy for all the studied beam sizes is shown in Fig. 3. It can be seen that the experimental data exhibit a marked upward trend, as previously determined from the description of the images in Fig. 1, and that there is a linear dependence of the volume of the transferred droplet on the laser pulse energy for all the analyzed beam sizes. Although the experimental points obtained with the smallest beam size also exhibit an upward trend, they show a slower rise than the others. A linear dependence of the ejected droplet volume on different technological parameters has been found for both piezoelectric ${ }^{26}$ and bubble jet ${ }^{27}$ printers. More specifically, in the latter case such a dependence has been found for the energy transferred to the liquid by the heating resistor. From the simple linear fit

$$
V=K\left(E-E_{0}\right)
$$

where $V$ is the droplet volume and $E$ is the laser pulse energy, the fitting parameters $K$ and $E_{0}$ were obtained and depicted in Table I. The proportionality constant $K$, which can be interpreted as the efficiency of the transfer process, presents a similar value for all the focusing conditions except the smallest beam radius, where $K$ is considerably smaller. The discrepancy in this latter case could be attributed to an underestimation of the droplet volume due to the splashing present in almost all of the analyzed rows (only the rows corresponding to the two lowest laser pulse energies considered in the graph are free from splashing); the error is more prominent the higher the laser pulse energy is. The obtained results also reveal that the other fitting parameter $\left(E_{0}\right)$ is higher the larger the dimensions of the laser beam are, thereby displaying a strong dependence on the focusing conditions. According to Eq. (1), $E_{0}$ would correspond to an energy threshold that must be overcome for a transfer to occur. However, it has to be pointed out that this is not a true transfer threshold, since higher laser pulse energies do not lead to a droplet transfer unless they achieve the minimum energy $E_{\text {min }}$, as noted previously. Finally, it is worth noting that the minimum droplet volume corresponding to each of the focusing conditions also displays a linear dependence on the minimum energy $E_{\text {min }}$ (Fig. 3).

In order to obtain a more comprehensive view of the transfer process, it would be appropriate to carry out a detailed inspection of the dimensional parameters related to both the laser beam and the ribbon that determine the final amount of the transferred liquid. Although a qualitative comparison of the lateral dimensions of both the liquid droplets and the corresponding laser spots has already been undertaken, from a quantitative point of view such comparison would not be adequate, since the radius of a droplet on a surface depends not only on the amount of liquid but also on the nature of the surface. As a first attempt, the easiest approach would involve looking for a correlation between the droplet volume and the volume of the liquid film delimited by the dimensions of the laser spot on the titanium coating, that is, a cylinder of elliptical section equal to the laser spot with a height equal to the thickness of the liquid film. Thus, the volume of the transferred droplets was plotted against the volume of the liquid film delimited by the elliptical area of the laser spot on the titanium coating, and the results are shown in Fig. 4. In general, the amount of the transferred liquid was considerably higher than the fraction of the liquid film lying above the zone corresponding to the laser spot. In fact, the previous finding that a droplet transfer was possible in the absence of ablation (experimental points on the vertical axis) already suggested that the dimensions of the laser spot do not delimit the amount of the ejected liquid. Never- 


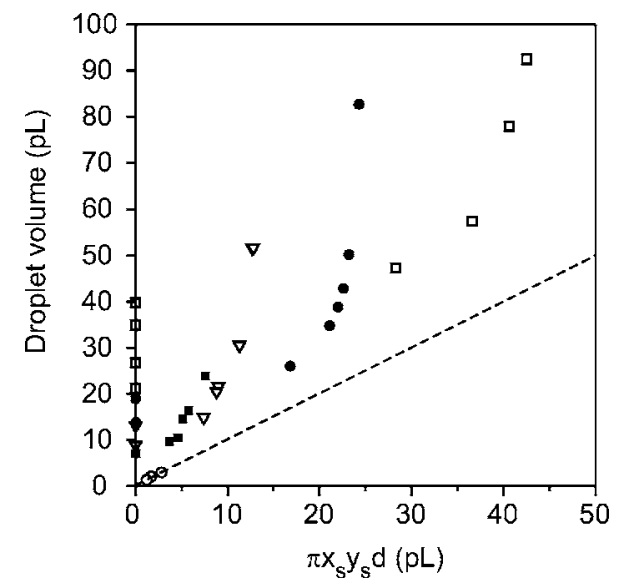

FIG. 4. Plot of the transferred droplet volume vs the volume of the liquid film delimited by the dimensions of the laser spot $\left(x_{s}\right.$ and $y_{s}$ correspond to the major and minor radii of the spot, respectively, and $d$ corresponds to the liquid film thickness) for all beam dimensions: $(\bigcirc) \omega_{x}=\omega_{y}=11 \mu \mathrm{m}$; ( $\square$ ) $\omega_{x}=31 \mu \mathrm{m}, \omega_{y}=25 \mu \mathrm{m} ;(\nabla) \omega_{x}=51 \mu \mathrm{m}, \omega_{y}=36 \mu \mathrm{m} ;(\bullet) \omega_{x}=73 \mu \mathrm{m}, \omega_{y}$ $=47 \mu \mathrm{m}$; and $(\square) \omega_{x}=95 \mu \mathrm{m}, \omega_{y}=64 \mu \mathrm{m}$. The dashed line represents the unity slope line.

theless, the plot in Fig. 4 was necessary since the behavior corresponding to the lowest energies for each of the focusing conditions could not be generalized to the whole energy range.

A second, more complex attempt to establish the dimensional parameter that determines the amount of the ejected liquid involves a more in-depth analysis of the threshold energy $E_{0}$ obtained from the linear fit corresponding to Eq. (1). A plot of $E_{0}$ versus the sectional area of the laser beam $\left(\pi \omega_{x} \omega_{y}\right)$ at the different focusing conditions (Fig. 5) reveals a clear proportionality dependence between these magnitudes. Taking into account that the local energy density of an elliptical Gaussian beam with energy $E_{0}$ is given by

$$
F(x, y)=\frac{2 E_{0}}{\pi \omega_{x} \omega_{y}} e^{-2\left(x^{2} / \omega_{x}^{2}+y^{2} / \omega_{y}^{2}\right)}
$$

where $x$ and $y$ are the Cartesian coordinates along the major and minor axes of the beam, respectively, the following relation holds:

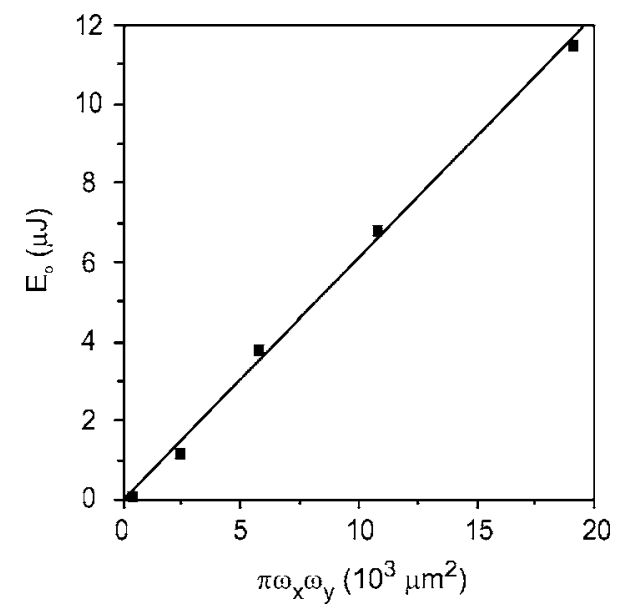

FIG. 5. Plot of the parameter $E_{0}$ vs the sectional area of the laser beam. The solid line corresponds to the linear fit.
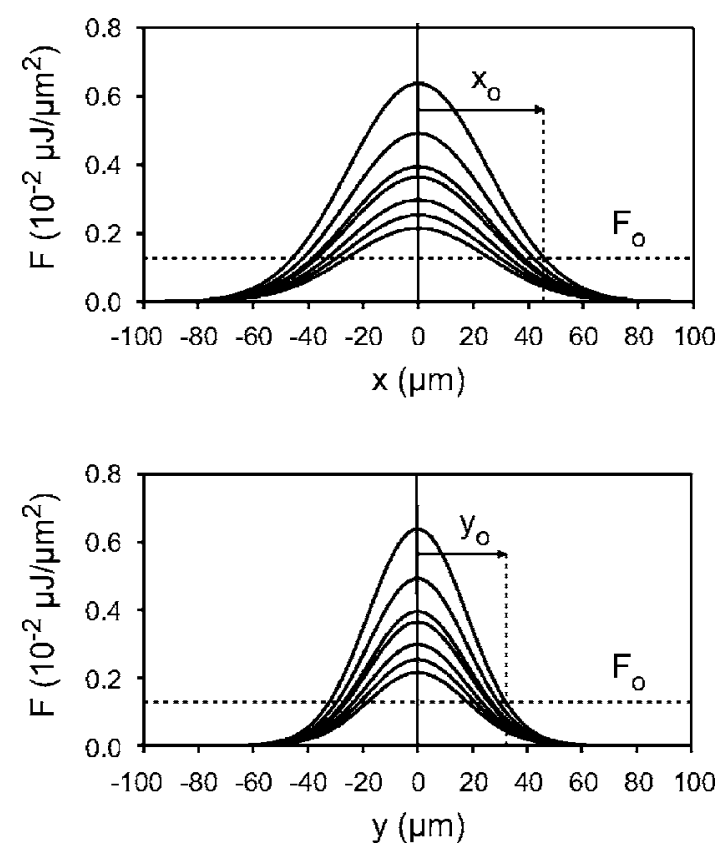

FIG. 6. Profiles of energy density distribution of the elliptical Gaussian beams corresponding to $\omega_{x}=95 \mu \mathrm{m}$ and $\omega_{y}=64 \mu \mathrm{m}$ for all the energies of Fig. 1(d). The intercepts with the threshold energy density $F_{0}$ determine the values of $x_{0}$ and $y_{0}$ (only those corresponding to the highest energy of $40 \mu \mathrm{J}$ are depicted).

$$
E_{0}=\frac{F(0,0)}{2} \pi \omega_{x} \omega_{y}=\frac{F_{0}}{2} \pi \omega_{x} \omega_{y}
$$

This indicates that all the Gaussian beams in Fig. 5 with energies equal to their own threshold energy $E_{0}$ have the same energy density peak $F_{0}$, and that $F_{0}$ therefore corresponds to an energy density threshold that is independent of the laser focusing conditions and is characteristic of the ribbon. The linear fit of the points in Fig. 5 provides a value for $F_{0}$ of $0.13 \mathrm{~J} / \mathrm{cm}^{2}$. It has been stated previously that the true energy threshold is not $E_{0}$ but, rather, $E_{\min }$. Thus, $F_{0}$ would be a threshold energy density in the sense that it would be a necessary but not sufficient condition for a transfer to occur. It could be interpreted as the energy density threshold required to produce a perturbation in the liquid film (generation of a vapor bubble or a mechanical wave, for instance), which would only be able to overcome the viscous and tension forces of the liquid and, therefore, eject a droplet, if the total energy delivered to the system reached the value $E_{\text {min }}$.

The parameter $F_{0}$ can now be used to determine the dimensions of the liquid zone delimited by the dimensions of such a perturbation, that is, the zone in which the local energy density $F(x, y)$ is higher than or equal to $F_{0}$. Certainly, for every Gaussian beam with an energy equal to or higher than $E_{\min }$, the major and minor radii $x_{0}$ and $y_{0}$, where the Gaussian distribution intercepts $F_{0}$, can be determined (Fig. 6 ). The volume of this zone would correspond to the cylinder of elliptical section $\pi x_{0} y_{0}$ and height equal to the liquid film thickness $d$. A plot of the droplet volume versus the cylinder volume for all the energies and laser focusing conditions is shown in Fig. 7. The points lie around the unity slope line, except those corresponding to the highest energies for each laser focusing condition, which lie well above. This indicates 


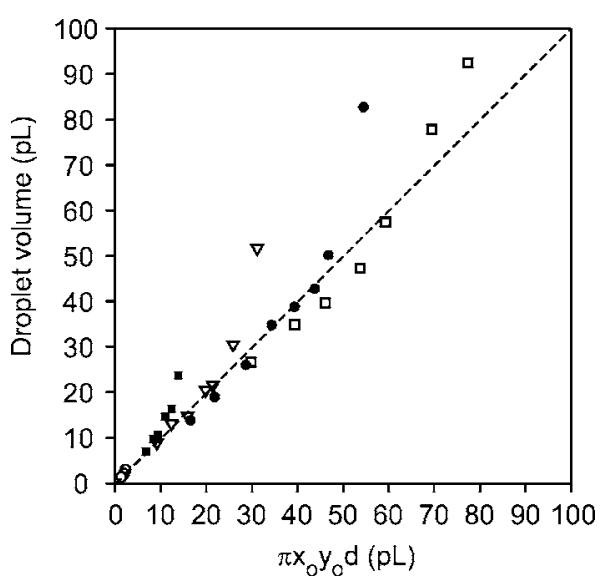

FIG. 7. Plot of the transferred droplet volume vs the volume of the liquid film delimited by the zone in which the energy density is higher than $F_{0}$ for all beam dimensions: (O) $\omega_{x}=\omega_{y}=11 \mu \mathrm{m}$; (ロ) $\omega_{x}=31 \mu \mathrm{m}, \omega_{y}=25 \mu \mathrm{m}$; $(\nabla) \omega_{x}=51 \mu \mathrm{m}, \omega_{y}=36 \mu \mathrm{m} ;(\bullet) \omega_{x}=73 \mu \mathrm{m}, \omega_{y}=47 \mu \mathrm{m}$; and ( $\left.\square\right) \omega_{x}$ $=95 \mu \mathrm{m}, \omega_{y}=64 \mu \mathrm{m}$. The dashed line represents the unity slope line.

that the amount of liquid removed by the action of the laser pulse is indeed determined by the area delimited by the threshold $F_{0}$. This statement only fails for pulse energies well above the minimum $E_{\min }$, where border effects become relevant: a significant lateral heat conduction could be responsible for the perturbation surpassing the dimensions imposed by $F_{0}$ or a significant liquid drag from the borders could occur, both at high laser pulse energies. Finally, it should be noted that the alignment of the experimental points in Fig. 7 around the unity slope line also indicates that the amount of the vaporized liquid is insignificant compared with the amount of the transferred material and that there is, therefore, almost no substantial material loss during the transfer process. This experimental result is consistent with the low heat penetration depth inside the liquid obtained with a simple estimation. ${ }^{18,28}$

\section{CONCLUSION}

The use of optical microscopy to evaluate microarrays prepared using LIFT revealed a dependence of the size, shape, and uniformity of the transferred droplets on the laser pulse energy and laser beam dimensions. The study showed that circular and uniform droplets are obtained with good reproducibility at laser pulse energies slightly above the transfer threshold energy for the specific laser beam dimensions. Thus, a precise control of the droplet diameter can be exerted through the simultaneous variation of the laser pulse energy and laser beam focusing conditions, without a loss in the quality of the droplet morphology. The smallest droplet diameter obtained is approximately $30 \mu \mathrm{m}$, a value well below those usually obtained with conventional microarray spotters.

The optical microscopy evaluation of the laser spots produced on the titanium coating after the transfer process showed that the ablation of the absorbing layer is not necessary for the transfer of liquid droplets by LIFT. The conditions in which the liquid transfer occurs without the removal of the coating correspond to those in which the smallest droplets with good morphological characteristics are obtained for each focusing condition.

Finally, quantitative analyses revealed that the droplet volume displays a linear dependence on the laser pulse energy for each focusing condition. These analyses also showed that the dimensional parameter that determines the amount of the transferred liquid per laser pulse is not the size of the laser spot on the titanium coating. Instead, there is an energy density threshold, independent of the laser focusing conditions, that acts as a necessary but not sufficient condition for a transfer to occur and that determines the volume of the liquid film ejected by the action of each laser pulse. The sufficient condition is given by a minimum energy threshold, which has a different value according to the specific laser beam dimensions. The last significant consequence of the analyses was that there is no marked material loss due to liquid vaporization during the transfer process.

\section{ACKNOWLEDGMENTS}

This work forms part of a research program funded by MEC of the Spanish Government (Project No. MAT200403741), the European Regional Development Fund (FEDER), and DURSI of the Catalan Government (2001 SGR00080).

${ }^{1}$ D. Rose, in Microarray Biochip Technology, edited by M. Schena (Eaton, Natick, 2000), p. 19.

${ }^{2}$ T. Okamoto, T. Suzuki, and N. Yamamoto, Nat. Biotechnol. 18, 438 (2000).

${ }^{3}$ J. Bohandy, B. F. Kim, and J. Adrian, J. Appl. Phys. 60, 1538 (1986)

${ }^{4}$ E. Fogarassy, C. Fuchs, F. Kerherve, G. Hauchecorne, and J. Perriére, J. Appl. Phys. 86, 457 (1989).

${ }^{5}$ Z. Kántor and T. Szörényi, J. Appl. Phys. 78, 2775 (1995).

${ }^{6}$ H. Esrom, J. Zhang, U. Kogelschatz, and J. Pedraza, Appl. Surf. Sci. 86, 202 (1995).

${ }^{7}$ P. K. Wu et al., Thin Solid Films 398/399, 607 (2001).

${ }^{8}$ B. R. Ringeisen, P. K. Wu, H. Kim, A. Piqué, R. Y. C. Auyeung, H. D. Young, and D. B. Chrisey, Biotechnol. Prog. 18, 1126 (2002).

${ }^{9}$ P. Serra, M. Colina, J. M. Fernández-Pradas, L. Sevilla, and J. L. Morenza, Appl. Phys. Lett. 85, 1639 (2004).

${ }^{10}$ I. Zergioti, A. Karaiskou, D. Papazoglou, C. Fotakis, M. Kapsetaki, and D. Kafetzopoulos, Appl. Phys. Lett. 86, 163902 (2005).

${ }^{11}$ J. M. Fernández-Pradas, P. Serra, M. Colina, and J. L. Morenza, Proc. SPIE 5662, 582 (2004)

${ }^{12}$ P. Serra, J. M. Fernández-Pradas, F. X. Berthet, M. Colina, J. Elvira, and J. L. Morenza, Appl. Phys. A: Mater. Sci. Process. 79, 949 (2004).

${ }^{13}$ M. Colina, P. Serra, J. M. Fernández-Pradas, L. Sevilla, and J. L. Morenza, Biosens. Bioelectron. 20, 1638 (2005).

${ }^{14}$ B. R. Ringeisen, D. B. Chrisey, A. Piqué, H. D. Young, R. Modi, M. Bucaro, J. Jones-Meehan, and B. J. Spargo, Biomaterials 23, 161 (2002).

${ }^{15}$ D. B. Chrisey, A. Piqué, R. A. McGill, J. S. Horwitz, B. R. Ringeisen, D. M. Bubb, and P. K. Wu, Chem. Rev. (Washington, D.C.) 103, 533 (2003).

${ }^{16}$ J. A. Barron, B. R. Ringeinsen, H. Kim, J. Spargo, and D. B. Chrisey, Thin Solid Films 453/454, 383 (2004).

${ }^{17}$ P. K. Wu et al., Rev. Sci. Instrum. 74, 2546 (2003).

${ }^{18}$ J. A. Barron, P. Wu, H. D. Ladouceur, and B. R. Ringeisen, Biomed. Microdevices 6, 139 (2004).

${ }^{19}$ J. A. Barron, R. Rosen, J. Jones-Meehan, B. J. Spargo, S. Belkin, and B. R. Ringeisen, Biosens. Bioelectron. 20, 246 (2004).

${ }^{20}$ B. Hopp, T. Smausz, N. Barna, Cs. Vass, Zs. Antal, L. Kredics, and D. Chrisey, J. Phys. D 28, 833 (2005).

${ }^{21}$ A. Piqué et al., Thin Solid Films 355/356, 536 (1999).

${ }^{22}$ P. Tran, D. A. Peiffer, Y. Shin, L. M. Meek, J. P. Brody, and K. W. Y. Cho, Nucleic Acids Res. 30, 54 (2002).

${ }^{23}$ P. Gong and D. Grainger, Surf. Sci. 570, 67 (2004). 
${ }^{24}$ D. C. D. Roux and J. J. Cooper-White, J. Colloid Interface Sci. 277, 424 (2004).

${ }^{25}$ D. Young, R. C. Y. Auyeung, A. Piqué, D. B. Chrisey, and D. Dlott, Appl. Phys. Lett. 78, 3169 (2001).
${ }^{26}$ N. Reis, C. A. Ainsley, and B. Derby, J. Appl. Phys. 97, 094903 (2005). ${ }^{27}$ A. Asai, T. Hara, and I. Endo, Jpn. J. Appl. Phys., Part 1 26, 1794 (1987).

${ }^{28}$ J. A. Barron, H. D. Young, D. D. Dlott, M. M. Darfler, D. B. Krizman, and B. R. Ringeisen, Proteomics 5, 4138 (2005). 